\title{
O modelo de atenção a saúde bucal no M édio e Baixo Xingu: parcerias, processos e perspectivas
}

\author{
The oral health care model in Middle and Low Xingu: \\ partnerships, processes, and perspectives
}

\author{
Pablo Natanael Lemos ${ }^{1}$ \\ Lucila Brandão H irooka ${ }^{1}$ \\ Selma A parecida Chaves N unes ${ }^{1}$ \\ Rui Arantes ${ }^{2}$ \\ Soraya Fernandes M estriner ${ }^{3}$ \\ Wilson M estriner Júnior ${ }^{3}$
}

${ }^{1}$ Departamento deM edicina Preventiva, Universidade Federal deSão Paulo. Rua Bacelar 346, Vila

Clementino. 04026-001 São Paulo SP.

pablonlemos@hotmail.com

2 Projeto Colgate Nativo.

${ }^{3}$ Departamento deClínica

Infantil, Odontologia

Preventiva e Social,

Faculdade de Odontologia

deRibeirão Preto,

UniversidadedeSão Paulo.
Abstract The purpose of this article is to present the actions developed to create the health care model at the Special Indigenous District - Xingu (DSEI-Xingu); particularly regarding oral health. An effective partnership established among thefollowing institutions U niversity Federal of StateSão Paulo, University of São Paulo at Ribeirão Preto College of Dentistry and Colgate ${ }^{\circledR}$, allowed the development of social health praxis at Middle and Low Xingu. The "Social Space" DSEI, which throughout the history, communities have developed and, through the social process of production, create differentiated accesses to consumer goods, is the basis for organizing health care services for the indigenous population. The DSEIXingu considers that establishing institutional partnerships as well as the effective participation of indigenous populations in health management in their territory is essential. Structured by plans based on population-reported problems, it uses the collective construction of explanatory networks, presenting solutions at different levels through an intersectorial approach. By observing health indicators, theindigenous communities' understanding of the recent primary health care model be comes perceptible, since it has been constantly applied to their culture, tradition, and uniqueness. Key words Indigenous health, $\mathrm{H}$ ealth care model, O ral health, Xingu Indigenous Park
Resumo Este artigo tem por objetivo apresentar as ações desenvolvidas na construção do model o de atenção em saúde no Distrito Especial Indígena Xingu (DSEI-Xingu), mais especificamente, na área de saúde bucal, com a efetiva parceria entrea UniversidadeFederal do Estado de São Paulo (UNIFESP), Faculdadede 0 dontologia de Ribeirão Preto - Universidade de São Paulo (FORP-USP) e a Colgate ${ }^{\circledR}$, que permitiu a construção social da práxisem saúdeno M édio eBaixo Xingu. Ao longo da história, o DSEI "Espaço Social" é onde as comunidades se constituem e, por meio do processo social de produção, cria acessos diferenciados aos bens de consumo, além de formar a base para a organização dos serviços de atenção à saúde dos povos indígenas. Para o DSEI-Xingu, são pontos básicos o estabelecimento de parcerias institucionais e a partici pação efetiva dos povos indígenas na gestão da saúde em seu território. Estruturado no planejamento baseado em problemas sentidos pela população, utiliza-se da construção coletiva de redes explicativas, apontando soluções em vários planos com abordagem intersetorial. É através da observação dos indicadores de saúde que se torna perceptível a assi milação das comunidades indígenas com o recente modelo de atenção básica à saúde bucal, uma vez queconstantementeestá sendo adaptado à cultura, à tradição e às singularidades desses povos indígenas.

Pal avras-chave Saúde indígena, M odel o deatenção, Saúde bucal, Parque Indígena do Xingu 
Introdução

A atenção básica constitui "um conjunto de saúde", no âmbito individual ou coletivo, que abrange a promoção e proteção à saúde, prevenção de agravos, diagnóstico, tratamento, reabilitação e a preservação da saúde, ações estas situadas no primeiro nível de atenção do sistema de saúde. A atenção básica édesenvolvida por meio do exercício de práticas gerenciais e sanitárias, democráticas e participativas, sob forma de trabalho em equipe, dirigidas a populações de territórios bem definidos, para as quais assumea responsabilidade sanitária, considerando a dinamicidade existente no território em que vivem essas populações ${ }^{1}$.

$\mathrm{Na}$ sua organização, um aspecto fundamental éo conhecimento do território, que não pode ser compreendido apenas como um espaço geográfico e delimitado para constituir a área deatuação dos serviços. Ao contrário, deve ser reconhecido como "Espaço Social” no qual, ao longo da história, a sociedade se constitui e por meio do processo social de produção cria acessos diferenciados aos bens de consumo, incluídos os serviços de saúde?.

A concepção trabalhada pela Universidade Federal de São Paulo (UNIFESP), em conjunto com povos indígenas na década de noventa, durante o movimento que levou à criação dos distritos sanitários, foi condizente com o conceito descrito acima, sendo que o compromisso com o processo social exige, até hoje, contínua refle xão. Estruturado no planejamento baseado em problemas sentidos pela população, permitiu a construção coletiva de redes explicativas, apontando soluções em vários planos com abordagem intersetorial, buscando garantir as especificidades necessárias à saúde dos povos indígenas ${ }^{3}$.

0 Parque Nacional do Xingu foi criado em 1961, em defesa de povos indígenas ameaçados pela venda de terras que ocupavam desde temposimemoráveise, no início da década de setenta, passou a ser denominado Parque Indígena do Xingu (PIX). De acordo com o Decreto $n^{\circ}$ 51.084, de 31 dejunho de 1961, da Presidência da República, o PIX tinha como um dos seus objetivos "garantir aos povos indígenas a assistência médica, social e educacional, indispensável para assegurar sua sobrevivência física e a preservação de seus atributos culturais"4.

O Distrito Sanitário Especial Indígena (DSEIXingu) foi instituído oficialmente em agosto de 1999, por meio de um convênio firmado pela Fundação Nacional de Saúde (FU NASA), gestora do subsistema de saúde indígena, e a UNI-
FESP, que passou a ser executora das ações de atenção básica à saúde. A decisão de estabelecer 0 convênio com a UNIFESP foi tomada por indicação das lideranças xinguanas.

\section{As parcerias}

Vários são os parceiros que vêm colaborando com o M inistério da Saúde na implantação dos Distritos Sanitários Especiais Indígenas como base para a organização dos serviços de atenção à saúde dos povos indígenas. São pontos básicos o estabelecimento de parcerias institucionais e a participação efetiva dos povos indígenas na gestão da saúde em seu território. É nesta perspectiva que o M édio e Baixo Xingu vêm desenvolvendo sua práxis em saúde. Mais especificamente, na área de saúde bucal, a efetiva parceria entre três instituições: UN IFESP, Faculdade de Odontologia de Ribeirão Preto-Universidade de São Paulo (FORP-USP) e Colgate ${ }^{\circledR}$ possibilitou a criação de um modelo de atenção em saúde bucal.

0 território do PIX está dividido em três áreas de abrangência, com sede em três polos-base: Pavuru, Diaurum e Wawi (Figura 1). Cada um deles possui uma equipe multiprofissional de saúdecompostapor médicos, enfermeiros, odontólogos, auxiliares indígenas de enfermagem (AIE) eagentes indígenas de saúde (AIS). A cada pólo são referenciadas al deias e local idades esuas respectivas populações (Quadro 1 ).

As ações de saúde propostas pelas parceiras estão previstas no Plano Distrital-PIX e, em consonância com a atual Política Nacional de Saúde Bucal $^{5}$, vêm sendo desenvolvidas por equipe multiprofissional contratada pela U NIFESP, que conta com a participação expressiva de egressos da FORP-USP. Atualmente, os dois cirurgiõesdentistas contratados pela U NIFESP para atuação permanente no DSEI-Xingu são egressos e participaram do estágio optativo da FORP-USP.

Além da prática tradicional de atendimento médico ea cobertura assistencial ininterrupta de equipes de saúdelocal, destacam-se ao longo dos anos o processo de formação técnica, universitária e de pós-graduação para profissionais de saúde, indígenas e não indígenas, e as perspectivas de construção social e conceitual de novas práticas de prevenção e promoção da saúde, na ótica da diversidade cultural.

Os objetivos específicos da área de saúde bucal da UNIFESP são a análise situacional, a formação de recursos humanos indígenas, a educação em saúde e a assistência odontológica. 


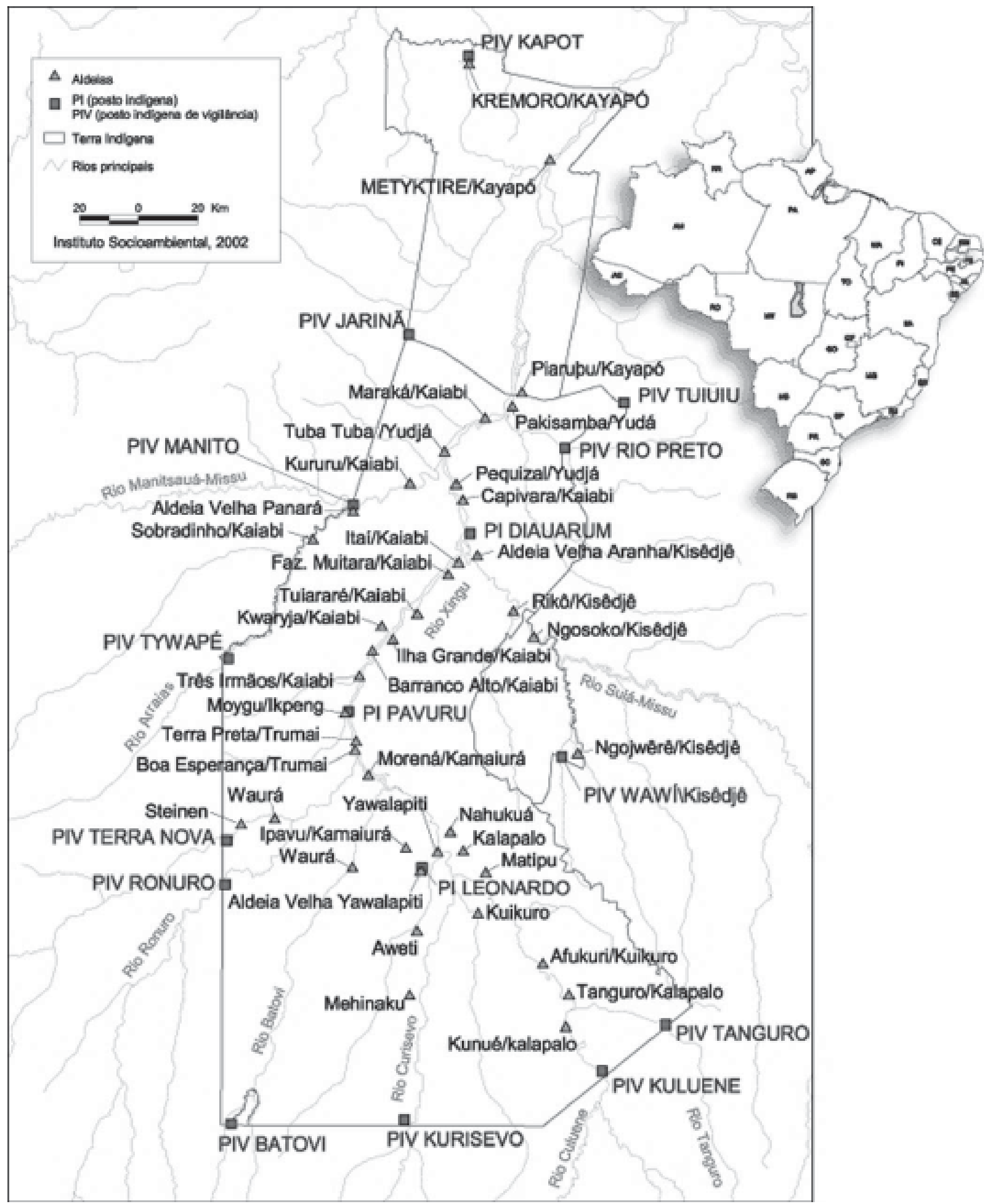

Figura 1. O Parque Indígena do Xingu - DSEI-Xingu ${ }^{11}$ - com as indicações dos quatro polos de saúde: Leonardo Villas Bôas, Pavuru, Diauarun e Wawi.

Fonte: Instituto Socioambiental ${ }^{6}$. 
Quadro 1. Etnias do Parque Indígena do Xingu - DSEI-Xingu - distribuídas segundo as aldeias e localidades assistidas e suas populações.

\begin{tabular}{|c|c|c|}
\hline Etnias / Família linguística & Aldeias & População \\
\hline KAIABI / Tupi & $\begin{array}{l}\text { Três Irmãos } \\
\text { Barranco Alto } \\
\text { Illha Grande } \\
\text { Guarujá } \\
\text { Tuiararé } \\
\text { M oitará } \\
\text { Itai } \\
\text { Piraquara } \\
\text { Capivara } \\
\text { Aiporé } \\
\text { Paranaíta } \\
\text { Fazenda M upada } \\
\text { Fazenda J oão } \\
\text { PIV M anito } \\
\text { Sobradinho } \\
\text { Maracá } \\
\text { Arraia }\end{array}$ & $\begin{array}{r}63 \\
31 \\
72 \\
89 \\
143 \\
35 \\
14 \\
06 \\
144 \\
67 \\
13 \\
13 \\
13 \\
24 \\
99 \\
35 \\
08\end{array}$ \\
\hline TRUMAI / I solada & $\begin{array}{l}\text { Boa Esperança } \\
\text { Terra Nova } \\
\text { Steinen } \\
\text { Cristalina }\end{array}$ & $\begin{array}{l}37 \\
42 \\
18 \\
15\end{array}$ \\
\hline SUIÁ / Jê & $\begin{array}{l}\text { N goiwere } \\
\text { Rapozão } \\
\text { Beira Rio } \\
\text { N gossoko }\end{array}$ & $\begin{array}{r}190 \\
27 \\
14 \\
44\end{array}$ \\
\hline JURUNA / Tupi & $\begin{array}{l}\text { Tuba-tuba } \\
\text { Parquissamba } \\
\text { Pequizal }\end{array}$ & $\begin{array}{r}149 \\
48 \\
31\end{array}$ \\
\hline WAURÁ / Arawak & Aruak & 45 \\
\hline KAM AIURÁ / Tupi & Morena & 96 \\
\hline IKPENG / Karibe & $\begin{array}{l}\text { Moigu } \\
\text { Ronuro }\end{array}$ & $\begin{array}{r}225 \\
25\end{array}$ \\
\hline IKPENG & * Polo Pavuru & 113 \\
\hline KAIABI- SUIÁ - JURUNA & ** Polo Diaurun & 307 \\
\hline SUIÁ & *** Polo Wawi & 4 \\
\hline TOTAL : 07 etnias & 31 aldeias / 03 polos-base & 2299 \\
\hline
\end{tabular}

Fonte: Dados da UN IFESP, 2006. 
M esmo contando com a grande experiência acumulada durante os quarenta anos do programa de saúde da U N IFESP no Parque I ndígena do Xingu, as instituições parceiras (Colgate ${ }^{\circledR}$ eFORPUSP) identificaram desde o início da ação conjunta a necessidade de conhecer o território onde iriam atuar, o queimplicou um processo de apropriação do espaço local e das relações da população, levando em consideração dados como perfil demográfico e epidemiológico, contexto histórico e cultural, reconhecimento das lideranças locais eoutros aspectos considerados relevantes para a intervenção no processo saúde-doença.

A seguir, as instituições desencadearam processos de mudança das práticas de saúde bucal, tornando-as mais adequadas aos problemas da realidade local.

Com a parceria firmada entre Colgate ${ }^{\circledR} \mathrm{e}$ UN IFESP, em 2003, o projeto Colgate Nativo se propôs desde seu início à redução da prevalência das doenças bucais e ao aumento da porcentagem de crianças de cinco a catorze anos com boas condições de saúde bucal, mediante o desenvolvimento de programas educativo-preventivos para a população, agentes indígenas de saúde e distribuição de insumos para higiene bucal (creme, escova efio dental).

$N$ a proposta inicial a atuação se dava em cinco aldeias: Waurá, M oigu, Tuiararé, N goiwere e Tuba-Tuba. No entanto, com a impossibilidade de continuar o projeto na aldeia Waurá, devido ao encerramento do convênio UN IFESP/FUNASA na área de abrangência do Alto Xingu, em 2004, a área de atuação do projeto foi redefinida para o M édio e Baixo Xingu.

Assim, foram criados núcleos de atuação que abrangem não apenas uma aldeia, mas um grupo de aldeias próximas e afins. Desta forma, ao invés de quatro aldeias-alvo, passou-se a ter quatro núcleos de atuação, a saber: núcleo Kaiabi (aldeias Tuiararé, Capivara, I lha Grande e Guarujá), núcleo I kpeng (Posto Pavuru eAldeia M oigu), núcleo Suiá (aldeias N goiwere, N gosoko, Beira Rio e Raposo), núcleo Yudjá (aldeias TubaTuba, Parque Samba e Pequizal).

A FORP-USP, em 2003, por convite feito pela UNIFESP, iniciou seu trabalho com o Projeto HUKA-KATU-USP ${ }^{7}$, contando com os recursos delogística einfraestrutura locais, disponibilizados pelas associações indígenas, FU N ASA eU N IFESP, complementado com recursos próprios previstos em seu protocolo de intenções.

Desdeo início, a FORP-USP apresentou a disposição de mobilizar recursos e apontar diretrizes para a inclusão das ações e equipes de saúde bucal, visando à extensão e universalização da cobertura assistencial, rotinização da atenção preventivo-promocional, com vistas à alteração do perfil dos problemas de saúde/doença dos povos da área abrangência do DSEI - Xingu.

Definindo a aldeia como local preferencial de realização das atividades acadêmicas de saúde bucal, desenvolve quatro entradas por ano, com duração aproximada de vinte dias, cobrindo em média cinco al deias por entrada. As ações expressam os princípios e diretrizes do SUS, da política Nacional deSaúde Bucal edas diretrizes curriculares para os cursos de saúde e apresentam as seguintes características operacionais: integralidade da assistência prestada à população no âmbito da atenção básica, com articulação da rede de comunicação entre os níveis de atenção; oferta de serviços de média e alta complexidade do SUS; humanização do atendimento e capacitação dos profissionais para trabalhar no contexto intercultural; abordagem multiprofissional; estímulo às ações de promoção de saúde, articulação intersetorial, participação e controle social e acompanhamento e avaliação permanente das ações realizadas.

Com a grande repercussão e boa avaliação feita pelas comunidades atendidas, no primeiro ano de sua atuação, a FORP-USP firmou, em outubro de 2004, a conviteda FUNASA, convênio com o Distrito Sanitário Especial Indígena do Xingu (DSEI - Xingu). E incluiu no seu novo currículo escolar o estágio optativo, que procura proporcionar aos estudantes o contato o mais cedo possível com a realidade social e serviços de saúde, a partir da observação e pelo desenvolvimento de atividades que dão condições ao estudante de superar a dicotomia entre estudo e trabalho.

Ressaltamos que, ao realizar esta atividade acadêmica, os estudantes são levados até a realidade da população assistida, aproximando-os do contexto sociocultural, o que permitea modificação da visão mecanicista e reducionista da natureza humana para uma concepção holística da vida. Esta experiência prática de ensino-aprendizagem tem permitido uma ampliação do referencial social ecultural do processo saúde-doença e suas implicações na prática odontológica.

\section{Processos eperspectivas}

São vários os produtos destas parcerias, sendo um dos principais a formação de recursos humanos na saúde indígena, com o enten dimento de que a efetivação das ações da atenção básica 
depende fundamentalmente de uma sólida política de educação permanente ecapaz de produzir profissionaiscom habilidadese competênciasque Ihes permitam compreender eatuar no SUS com competência técnica, espírito crítico e compromisso político em saúde.

Assegurar 0 acesso dos povos indígenas às ações dos povos indígenas no DSEI-Xingu tem sido uma preocupação constante, respeitando e valorizando suas práticas tradicionais, além de respeitar os limites da ética profissional. Em quatro anos, o programa de saúde bucal alcançou as seguintes metas:

- propiciou a incorporação de procedimentos coletivos e individuais em locais onde não há consultórios odontológicos;

- adequou à prática de controle de infecção intrabucal nas aldeias;

- propiciou uma ação integrada com as áreas da saúde e autilização de diversos espaços sociais para o desenvolvimento de ações coletivas desaúde bucal;

. consolidou a prática efetiva de discussão local com a população indígena sobre as ações desenvolvidas;

- utilizou a epidemiologia como instrumento organizador da atenção;

- atuou em plena consonância com os AIS (agente indígena de saúde), buscando a melhoria da qualidade e do acesso às atividades em saúde bucal;

. organizou a demanda assistencial a partir do levantamento denecessidades ( programação).

Quanto à avaliação em saúde, por ser um processo crítico-reflexivo sobre práticas e processos desenvolvidos no âmbito dos serviços de saúde, deve ser contínua esistemática esua temporalidade é definida em função do âmbito em que ela se estabelece. A avaliação não deve ser exclusivamente um procedimento de natureza técnica, embora essa dimensão esteja presente, devendo ser entendida como processo denegociação entre atores sociais. Por esta razão, a avaliação é desenvolvida constantemente de forma a permitir a negociação e pactuação entre sujeitos que partilham corresponsabilidades ${ }^{8}$.

$\mathrm{Na}$ prática, as informações epidemiológicas são sempre difíceis deserem dimensionadas para os povos indígenas (que figuram como denominador do indicador), pois, via de regra, são de baixa população, o que faz com que pequenas variações no denominador impliquem alterações do indicador e dificultem sua interpretação. Em resposta a esse desafio, as parceiras estão mobilizadas para a tabulação dos resultados do le- vantamento epidemiológico das condições de saúdebucal no DSEI-Xingu, mesmo considerando que o quadro epidemiológico dos povos indígenas é marcado por uma escassez de dados que tende a inviabilizar o conhecimento de um perfil epidemiológico amplo e robusto. 0 senso comum é o de que os impactos decorrentes do contato com a sociedade nacional, sobretudo nas formas de subsistência, envolvendo mudanças na dieta com a entrada de alimentos industrializados e do açúcar refinado, repercutem negativamente na saúde bucal. Finalmente, de nossa parte, é de se esperar que o conhecimento do quadro epidemiológico contribua com o esforço nacional voltado para o estudo das condições de saúde dos povos indígenas e para criação e manutenção de uma base eletrônica de dados relativa aos principais problemas, contribuindo inclusive para a estruturação de um sistema nacional de vigilância epidemiológica em saúde bucal.

Para a ampliação das ações em desenvolvimento, as instituições parceiras têm incorporado constantementetecnologias deelevada complexidade (baixa densidade), no intuito deatender aos problemas de saúde de maior frequência e relevância em sua área de ação, tendo como orientação os princípios da universalidade, da acessibilidade e da coordenação do cuidado, do vínculo e continuidade, da integralidade, da responsabilização, da humanização, da equidade e da participação social.

Concordamos com D ouglas Rodrigues ${ }^{9}$ quanto à possibilidade deutilização dos indicadores de saúde clássicos comparados no tempo e no espaço, ao lado de indicadores elaborados especialmente, contemplando as características eespecificidades de cada projeto, não havendo oposição entre indicadores clássicos da saúde pública, indicadores específicos de cada projeto, organização ou contextos específicos e indicadores relativos às concepções, ideários e perspectivas coletivas.

As parceiras têm se empenhado na obtenção de alguns indicadores de saúde bucal definidos por representação numérica e que consideram a observação de determinados eventos (atividades realizadas, ocorrência de doenças) (Q uadro 2).

As informações permitem 0 conhecimento (quantitativo ou qualitativo) de uma determinada situação, com o propósito de tomar decisões e agir, com o objetivo de transformar a realidade compreen dida no espaço indicado. Passariam a ser ferramentas importantes para avaliação da gestão, para a aferição da eficiência e da eficácia de interven ções de projetos de modificação da realidade no setor saúde. 
Quadro 2. Evolução dos indicadores de saúde bucal no DSEI-Xingu, 2004, 2005, 2006.

\begin{tabular}{|c|c|c|c|c|}
\hline Indicadores & D escrição & 2004 & 2005 & 2006 \\
\hline Acesso & $\begin{array}{l}\text { Exames clínicos / população total da } \\
\text { área x } 100\end{array}$ & $61,05 \%$ & $89,57 \%$ & $117,54 \%$ \\
\hline $\begin{array}{l}\text { Controle doença cárie e } \\
\text { doença periodontal }\end{array}$ & $\begin{array}{l}\text { Indivíduos com } 1^{\text {a }} \text { fase de } \\
\text { tratamento concluído* / população } \\
\text { total da área x } 100\end{array}$ & $24,39 \%$ & $41,97 \%$ & $38,33 \%$ \\
\hline $\begin{array}{l}\text { Relação restaurações/ } \\
\text { extrações }\end{array}$ & $\begin{array}{l}\text { Total restaurações /total de } \\
\text { extrações }\end{array}$ & 2,31 & 5,08 & 5,12 \\
\hline Capacitação AIS & $\begin{array}{l}\text { Total de AISB participantes dos } \\
\text { cursos/total de AISB x } 100\end{array}$ & - & $87,59 \%$ & $100 \%$ \\
\hline Promoção de saúde & $\begin{array}{l}\text { Total de participantes dos eventos } \\
\text { de educação / população total da } \\
\text { área x } 100\end{array}$ & - & $44,40 \%$ & $39,71 \%$ \\
\hline Ações coletivas & $\begin{array}{l}\text { Total de participantes da escovação } \\
\text { supervisionada e ATF / população } \\
\text { total da área x } 100\end{array}$ & - & $95,48 \%$ & $163,85 \%$ \\
\hline
\end{tabular}

*Adequação do meio bucal.

Já no tratamento reabilitador, desenvolvido no período a que se refere 0 artigo, foram confeccionadas um total de 313 próteses reabilitadoras (PT-PPR) com 171 pessoas beneficiadas, de um total de 1.150 indivíduos com mais de quinze anos de idade, correspondendo aproximadamente a $70 \%$ da população que necessita de próteses reabilitadoras.

Pela observação dos indicadores, já se torna perceptível a assimilação das comunidades indígenas ao recente modelo de atenção básica à saúde bucal, que a cada dia está sendo adaptado à cultura, à tradição e às singularidades desses povos indígenas.

As parcerias possibilitaram a horizontalização das ações de saúde bucal e a sua integração com outras áreas da saúde. A integralidade na atenção não se limitou ao simples atendimento à demanda espontânea, mas desenvolveu meios de sensibilizar no indivíduo a importância das ações coletivas de ampla resolução. N esta prática, foram envolvidos estudantes das instituições conveniadas, profissionais de saúde, agentes indígenas de saúde e, até mesmo, professores que atuam nas comunidades indígenas e lideranças locais.
O modelo de atenção não introduziu apenas novas tecnologias para o tratamento da doença cáriee da doença periodontal, mas sim uma nova filosofia e conceitos: as ações de promoção de saúde são descentralizadas emultiplicativas, uma vez que são compartilhadas com agentes indígenas de saúde, professores, pajés, pais e outros, sob a responsabilidade e a orientação da equipe. N ossa principal ponte de ligação, em razão da linguagem, abordagem e convivência são os agentes indígenas de saúde bucal, que são pessoas da comunidade.

As ações coletivas e individuais promoveram a interação dos comunitários com as equipes de saúde bucal através de reuniões, palestras, oficinas de avaliação dos serviços de saúde, atenção individualizada, distribuição de escovas edentifrícios, práticas de escovação ecapacitação dos agentes indígenas de saúde (AIS) e agentes indígenas de saúde bucal (AISB), que são os pilares do processo de educação continuada nas comunidades.

A formação dos indígenas para o trabalho em saúde foi estratégia fundamental para a construção do DESI-Xingu, pois teve como princípios a aprendizagem em serviço, dentro da realidade do 
território indígena, ea concepção de prática sanitária baseada no modelo da vigilância da saúde ${ }^{10}$.

E, na construção do modelo, por ser processo social, esta situação é única, singular, histórica, não passível de replicação ou reprodução mecânica em qualquer outra situação concreta, uma vez que os elementos e dimensões de cada

\section{Colaboradores}

PL Lemos, LB Hirooka, SAC Nunes, R Arantes, SF Mestriner e W Mestriner Jr participaram igualmente de todas as etapas da elaboração do artigo.

\section{Referências}

1. Brasil. Ministério da Saúde. Secretaria de Atenção Básica à Saúde. Departamento de Atenção Básica. Cadernos da Atenção Básica n. 17: Saúde Bucal. Brasília: M inistério da Saúde; 2006.

2. Mendes EV. Distrito Sanitário: 0 processo social de mudança das práticas sanitárias do SUS. São Paulo: Hucitec; Rio de Janeiro: Abrasco; 1995.

3. Baruzzi RG, Junqueira C. Parque Indígena do Xingu: saúde, cultura e história. São Paulo: Terra Virgem; 2005.

4. Brasil. Decreto no 51.084, de 31 de julho de 1961. Regulamenta o Decreto no 50.455, de 14 de abril de 1961, que criou o Parque Nacional de Xingu, e dá outras providências. Diário Oficial da União 1961; 8 ago.

5. Brasil. Ministério da Saúde. Secretaria de Atenção Básica à Saúde. Departamento de Atenção Básica. Coordenação Nacional de Saúde Bucal. Diretrizes da Política Nacional de Saúde Bucal. Brasília: M inistério da Saúde; 2004.

6. Instituto Socioambiental. Enciclopédia. Parque Indígena do Xingu [Mapa] 2002. [site da Internet] [acessado 2007 ago 16]. Disponível em: http://www. socioambiental.org/pib/epi/xingu/parque.shtm

7. Oliveira C, Pacagnella RC, Chiarello MGM, Bregagnolo JC, Watanabe M GC, M estriner JW. "Proje to Huka Katu: a FORP - USP no Parque Indígena do Xingu". Rev ABENO 2005; 5(2):135-139. um desses processos apresentam contradições, geram conflitos e são marcados por negociações e pactos que Ihes são próprios, específicos.

Por isto, a saúde bucal das populações xinguanas "não resulta apenas da prática odontológica, mas deconstruções sociais operadas demodo consciente pelos homens, em cada situação concreta" ${ }^{11}$.
8. Brasil. Ministério da Saúde. DATAUnB - Pesquisas Sociais Aplicadas. Centro de Pesquisas sobre Opinião Pública da Universidade de Brasília. Pesquisa Nacional de Avaliação da Satisfação dos Usuários do SUS. [site da Internet] 2006 [acessado 2007 jun 30]; [cerca de 16 p.]. Disponível em: http://portal.saude. gov.br/portal/arquivos/pdf/resumo_do_projeto_ satisfacao_usuarios_sus.pdf

9. Rodrigues ${ }^{-}$D. Indicadores de Saúde: uso em populações indígenas. In: Fórum de ONGs. Associação Saúde sem Limites e a H ealth Unlimited; 2003; São Paulo.

10. Teixeira C, Paim J, Villasboas AL. SUS, Modelos Assistenciais e Vigilância da Saúde. Inf epidemiol SUS 1998; 7(2):7-28.

11. Narvai PC, Frazão P. Epidemiologia, política, e saúde bucal coletiva. In: Antunes JLF, Peres MA, organizadores. Epidemiologia da saúde bucal. Rio de Janeiro: Guanabara Koogan; 2006. p. 346-362.
Artigo apresentado em 19/05/2007 Aprovado em 14/12//2007 